\title{
A LITERATURE REVIEW ON THE VEHICLE ROUTING PROBLEM WITH MULTIPLE DEPOTS
}

\begin{abstract}
In this paper, we present a state-of-the-art survey on the vehicle routing problem with multiple depots (MDVRP). Our review considered papers published between 1988 and 2014, in which several variants of the model are studied: time windows, split delivery, heterogeneous fleet, periodic deliveries, and pickup and delivery. The review also classifies the approaches according to the single or multiple objectives that are optimized. Some lines for further research are presented as well.
\end{abstract}

